\title{
Left atrial Appendage Rupture Following Intraoperative Hypertensive Crisis
}

\author{
Mehrab Marzban $^{1}$ and Peyman Benharash ${ }^{2}$ \\ ${ }^{1} \mathrm{UCLA}$ \\ ${ }^{2}$ University of California, Los Angeles
}

January 8, 2022

\begin{abstract}
The acute and severe rise in systemic blood pressure during or after cardiac operations may be life-threatening and result in end-organ injury. This case is the first report of spontaneous left atrial appendage rupture following the hypertensive crisis in cardiac surgery
\end{abstract}

Title page

Left atrial Appendage Rupture Following Intraoperative Hypertensive Crisis

Mehrab Marzban, MD. Peyman Benharash,MD

Cardiovascular Outcomes Research Laboratories, Division of Cardiac Surgery, David Geffen School of Medicine at UCLA, Los Angeles, California

Corresponding author: Mehrab Marzban,MD

Email:MMarzban@mednet.ucla.edu

10833 Le Conte Ave.62-249 CHS

Los Angeles, CA 90095

Tel:(310)206-6717

Abstract:

The acute and severe rise in systemic blood pressure during or after cardiac operations may be life- threatening and result in end-organ injury. This case is the first report of spontaneous left atrial appendage rupture following hypertensive crisis in cardiac surgery

Introduction: Hypertension is the leading cause of mortality and morbidity worldwide. $(1,2)$ It is estimated that nearly half of all cardiovascular diseases are attributable to uncontrolled hypertension. (3) Intraoperative hypertension in cardiovascular operations may result in bleeding that may be diffuse or related to suturelines, resulting in retained hematoma and occasionally re-exploration. Moreover, high blood pressure may result in acute aortic dissection, acute pulmonary edema, kidney injury, or intracranial hemorrhage.

Case presentation: A waiver for the case report was obtained from the Hospital Ethics committee. An 84-year- old woman with a long standing history of hypertension and hyperlipidemia presented with stable angina and was found to have three vessel coronary artery disease including the left main. Preoperative echocardiography showed a left ventricular ejection fraction of $45 \%$ with inferobasal hypokinesis and moderate 
ischemic mitral regurgitation. She underwent on-pump coronary artery bypass grafting (CABG) $\mathrm{x} 4$ through a conventional approach utilizing the left internal mammary artery and saphenous vein grafts. She was easily weaned from cardiopulmonary bypass and decannulated. Following routine hemostasis and sternal closure, the patient became hypertensive with blood pressures as high as $165 / 90 \mathrm{mmHg}$. In attempts to reduce the blood pressure, the anesthesiologist inadvertently administered a bolus of epinephrine, resulting in severe tachycardia and hypertension up with systolic blood pressures of nearly $240 \mathrm{mmHg}$. Shortly thereafter, the patient developed marked bright red bleeding from mediastinal drains. Upon re-exploration, bleeding was noted from behind the heart. After lifting the heart, a punctate site at the tip of the left atrial appendage (LAA) was found to be the source which was repaired with a 4-0 prolene suture (fig 1A) Distal and proximal anastomosis suture lines were hemostatic and free of bleeding. Intraoperative trans esophageal echocardiography revealed near normal wall motion without evidence of dissection in heart chambers or ascending aorta. (fig 2) Although the patient's postoperative course was complicated by delirium, she recovered completely and was discharged home on the 6th postoperative day.

Comment: To our knowledge, this is the first reported case of spontaneous left atrial appendage rupture during cardiac surgery. Prior work has described both early and late tamponade following percutaneous device closure of the LAA $(5,6$,$) . From a surgical standpoint, the LAA auricle may be caught in the jaws$ of the aortic cross clamp, causing iatrogenic injury. (7) This complication is generally a point of concern with the limited view in minimally invasive operations when the cross clamp may injure the LAA through the transverse sinus. To avoid this complication, it is crucial to visualize the tips of the cross-clamp during its application. Acknowledging this complication, we always lift the aorta under reduced bypass flow for cross clamping and routinely we check the clamp location. Although the exact mechanism of injury was not clear in the present case, it can be assumed that a micro perforation or crushing of the LAA had occurred. Following the operation, the severe rise in left ventricular pressure was likely transmitted to the left atrium through a regurgitant mitral valve. Left atrial dissection is another rare complication that can be the cause of unexplained hematoma or hemorrhage usually during valve operations. Its presence was excluded in our case by the general appearance of the appendage and via transesophageal echocardiography (Figure 1B). Technical considerations aside, this case illustrates the catastrophic consequences of medication errors in the operating room. Such errors may occur at any stage and may result from name confusion, prescription error and errors in dosage or route of administration. (8)

Our patient and us were lucky that she did not suffer permanent damage. Had this complication occurred in the postoperative period, the chances of saving the patient without any neurological injury would have been remote.

References:

1. Lopez AD, Mathers CD, Ezzati M, Jamison DT, Murray CJ. Global and regional burden of disease and risk factors, 2001: systematic analysis of population health data. Lancet. 2006;367:1747-1757 .

2.Donghao Zhou, Bo Xi, Min Zhao, Liang Wang, Sreenivas P. Veeranki

Uncontrolled hypertension increases risk of all-cause and cardiovascular disease mortality in US adults: the NHANES III Linked Mortality Study. Sci Rep. 2018; 8: 9418.

3. Lawes CM, Vander Hoorn S, Rodgers A. International Society of Hypertension. Global burden of bloodpressure-related disease, 2001. Lancet. 2008;371:1513-1518.

\section{George A Mensah 1, Janet B Croft, Wayne H Giles}

The heart, kidney, and brain as target organs in hypertension. Cardiol Clin. 2002 May;20(2):225-47

5.Jian-Ming Wang, Qi-Guang Wang, and Xian-Yang Zhu. Delayed cardiac tamponade after simultaneous transcatheter atrial septal defect closure and left atrial appendage closure device implantation: a particular case report. J Geriatr Cardiol. 2019 Dec; 16(12): 898-901. 
6.Karen P. Phillips, Christopher Smith, Michelle Butler, Anders Taylor, Terri Hall, Epicardial appendage ooze causing pericardial tamponade after left atrial appendage device implantation

HeartRhythm Case Rep. 2018 Aug; 4(8): 350-352.

7.Markus Czesla, Julia Götte, Timo Weimar, Tamas Ruttkay, and Nicolas Doll. Safeguards and pitfalls in minimally invasive mitral valve surgery. Ann Cardiothorac Surg. 2013 Nov; 2(6): 849-852.

8. Medication Errors in Acute Cardiac Care. Circulation. 2002;106:2623-2629

Fig.1.Operative view; punctate rupture of LAA

Fig.2.Intraoperative TEE: No evidence of LAA dissection

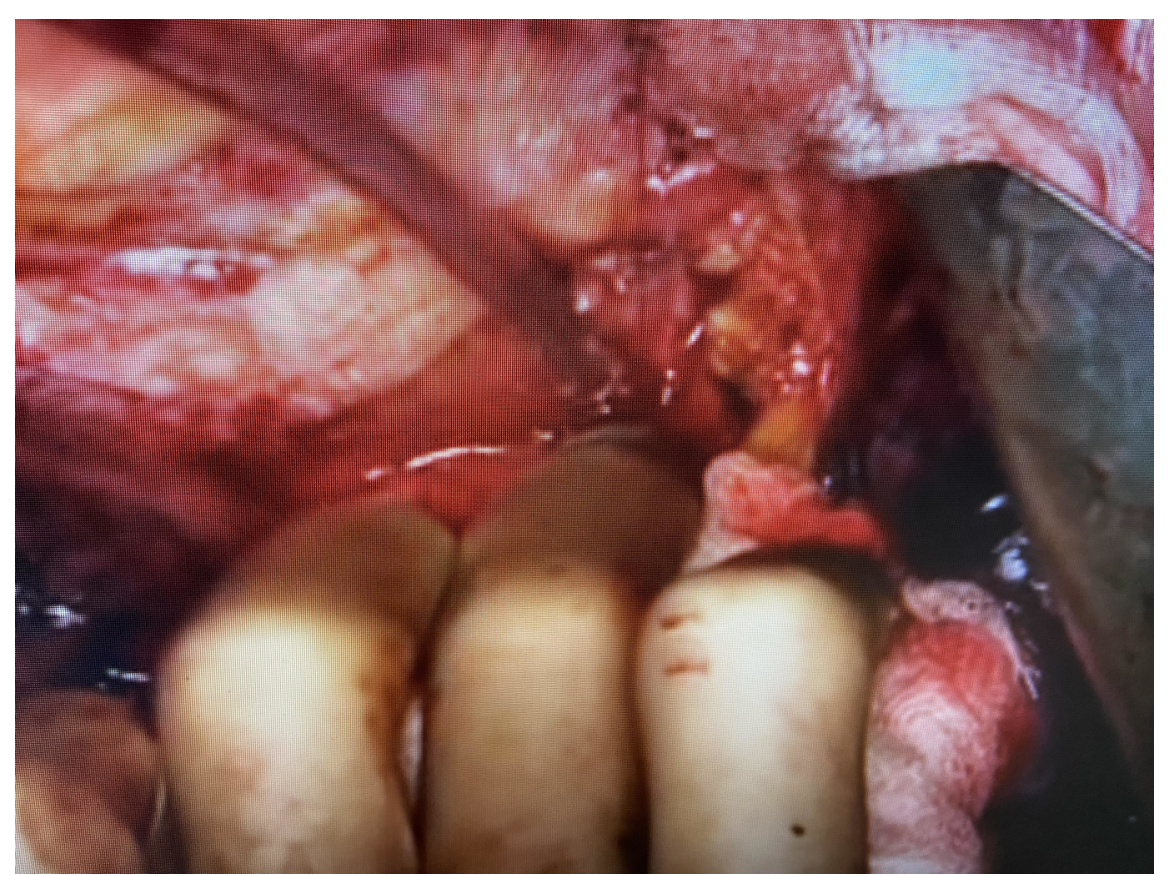




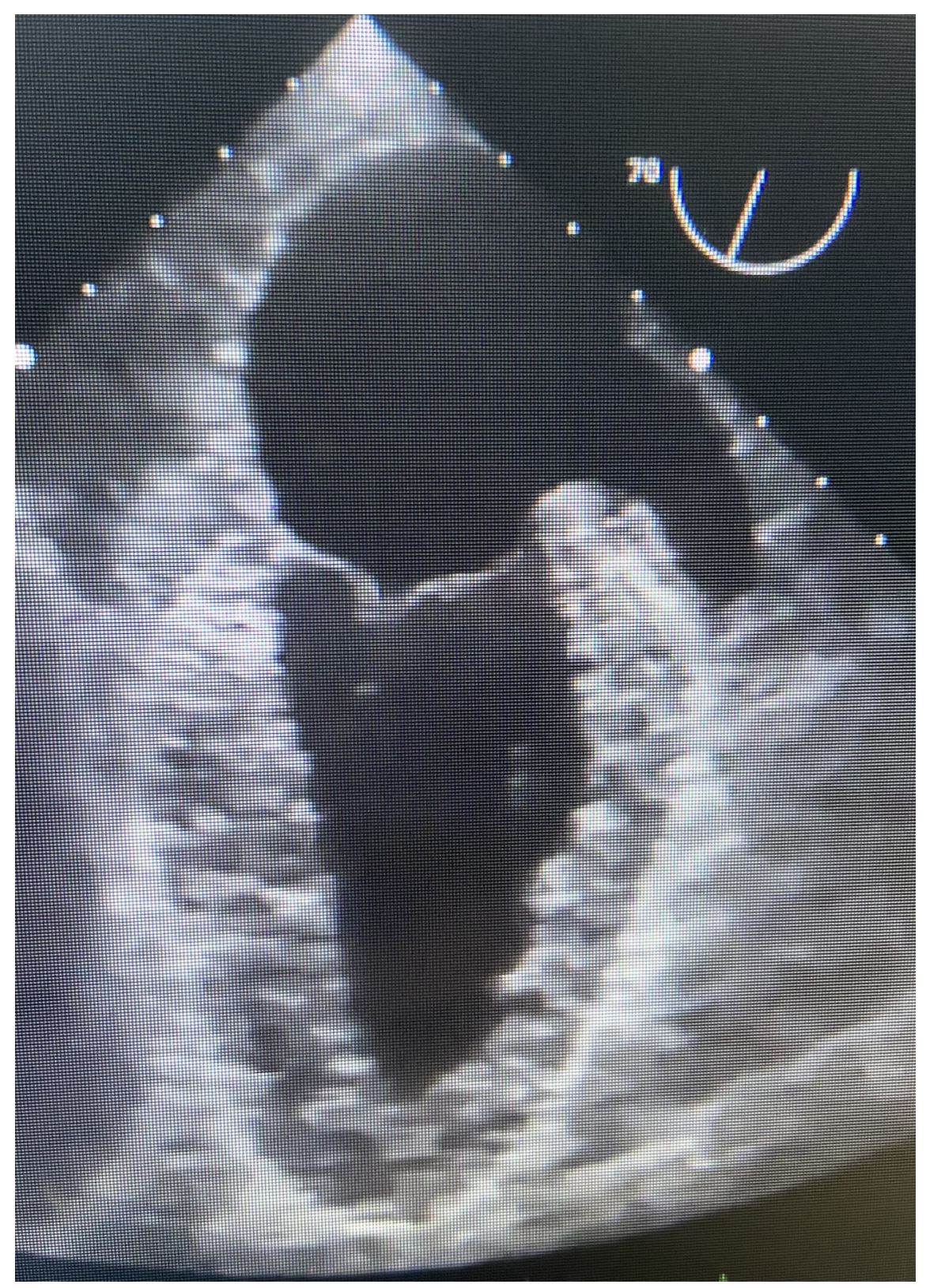

